\title{
COMMUTATIVITY THEOREMS FOR RINGS WITH CONSTRAINTS ON COMMUTATORS
}

\author{
H. A. S. ABUJABAL AND MOHD. ASHRAF
}

\begin{abstract}
Let $R$ be a left (resp. right) $s$-unital ring and $m$ be a positive integer. Suppose that for each $y$ in $R$ there exist $f(t), g(t), h(t)$ in $Z[t]$ such that $x^{m}[x, y]=$ $g(y)\left[x, y^{2} f(y)\right] h(y)$ (resp. $\left.[x, y] x^{m}=g(y)\left[x, y^{2} f(y)\right] h(y)\right)$ for all $x$ in $R$. Then $R$ is commutative (and conversely). Finally, the result is extended to the case when the exponent $m$ depends on the choice of $x$ and $y$.
\end{abstract}

\section{Introduction}

Throughout the paper, $R$ will denote an associative ring (may be without unity 1 ), and for any $x, y$ in $R$ the symbol $[x, y]$ stands for the commutator $x y-y x$. A ring $R$ is called left (resp. right) $s$-unital if $x \in R x$ (resp. $x \in x R$ ) for all $x$ in $R$, and $R$ is called $s$-unital if $x \in R x \cap x R$ for all $x$ in $R$. As usual $\mathbb{Z}[t]$ is the totality of polynomials in $t$ with coefficients in $\mathbb{Z}$, the ring of integers. We consider the following ring properties:

$\left(P_{1}\right)$ For each $y$ in $R$ there exist $f(t), g(t), h(t)$ in $\mathbb{Z}[t]$ such that $x^{m}[x, y]=g(y)[x$, $\left.y^{2} f(y)\right] h(y)$ for all $x$ in $R$, where $m$ is a fixed positive integer.

$\left(P_{1}^{*}\right)$ For each $x, y$ in $R$ there exist a positive integer $m$ and $f(t), g(t), h(t)$ in $\mathbb{Z}[t]$ such that $x^{m}[x, y]=g(y)\left[x, y^{2} f(y)\right] h(y)$.

$\left(P_{2}\right)$ For each $y$ in $R$ there exist $f(t), g(t), h(t)$ in $\mathbb{Z}[t]$ such that $[x, y] x^{m}=g(y)[x$, $\left.y^{2} f(y)\right] h(y)$ for all $x$ in $R$, where $m$ is a fixed positive integer.

$\left(P_{2}^{*}\right)$ For each $x, y$ in $R$ there exist a positive integer $m$ and $f(t), g(t), h(t)$ in $\mathbb{Z}[t]$ such that $[x, y] x^{m} \doteq g(y)\left[x, y^{2} f(y)\right] h(y)$.

(CH) For each $x, y$ in $R$ there exist $p(t), q(t) \in t^{2} \mathbb{Z}[t]$ such that $[x-p(x), y-q(y)]=0$.

A. well-known theorem of Bell [5] states that if $n>1$ is a positive integer and $R$ is an $n$-torsion free ring with unity 1 such that $\left[x^{n}, y\right]=\left[x, y^{n}\right]$ for all $x, y$ in $R$,

Received September 3, 1993; revised February 22, 1994.

1991 Mathematics Subject Classification. 16U80.

Key words and phrases. s-unital rings, factor subrings, polynomial identity, semiprime rings, commutators. 
then $R$ is commutative. Recently, Psomopoulos [11] generalized the above result and proved that if $R$ is an $n$-torsion free ring with unity 1 satisfying the polynomial identity $x^{t}\left[x^{n}, y\right]=\left[x, y^{m}\right] y^{s}$ for non-negative integers $m \geq 1, n>1, t \geq 0, s \geq 0$, then $R$ is commutative. In the present paper our objective is to further extend the later result to rings satisfying condition of the form $\left(P_{1}\right)$ or $\left(P_{2}\right)$. We shall also investigate the commutativity of rings satisfying either of the properties $\left(P_{1}^{*}\right)$ and $\left(P_{2}^{*}\right)$ together with the Chacron's condition $(\mathrm{CH})$.

\section{Some preliminary results}

We being by considering the following types of rings:
(a) $\left(\begin{array}{cc}G F(p) & G F(p) \\ 0 & G F(p)\end{array}\right), p$ a prime.
$(a)_{1}\left(\begin{array}{cc}G F(p) & G F(p) \\ 0 & 0\end{array}\right), p$ a prime.
$(a)_{r}\left(\begin{array}{ll}0 & G F(p) \\ 0 & G F(p)\end{array}\right), p$ a prime. (b) $M_{\sigma}(K)=\left\{\left(\begin{array}{cc}\alpha & \beta \\ 0 & \sigma(\alpha)\end{array}\right) / \alpha, \beta \in K\right\}$, where $K$ is a finite field with a non-trivial auto-
morphism $\sigma$.

(c) A non-commutative ring with non-zero divisors of zero.

(d) $S=\langle 1\rangle+T, T$ a non-commutative subring of $S$ such that $T[T, T]=[T, T] T=0$.

In a paper [12], Streb gave a classification for non-commutative rings, which has been used effectively to obtain a number of commutativity theorems (cf. [8], [9] \& [10]). Further, from the proof of [12, Corollary (1)] one can easily observe that if $R$ is a noncommutative left $s$-unital ring, then there exists a factor subring of $R$ which is of type $(a)_{1},(b),(c)$ or $(d)$. This result gives the following lemma which plays the key role in our subsequent study (cf. [9, Meta Theorem]).

Lemma 1. Let $P$ be a ring property which is inherited by factor subrings. If no rings of type $(a)_{1}$ (resp. $\left.(a)_{r}\right),(b),(c)$ or $(d)$ satisfy $P$, then every left (resp. right) s-unital satisfying $P$ is commutative.

The proofs of the following lemmas can be found in [7], [8] and [10] respectively.

Lemma 2. Let $f$ be a polynomial in non-commuting indeterminates $x_{1}, x_{2}$, $\ldots, x_{n}$ with relatively prime integer coefficients. Then the following are equivalent.

(i) Every ring satisfying the polynomial identity $f=0$ has nil commutator ideal.

(ii) Every semi-prime ring satisfying $f=0$ is commutative.

(iii) For every prime $p,(G F(p))_{2}$ fail to satisfy $f=0$.

Lemma 3. Suppose that a ring $R$ with unity 1 satisfies $(C H)$. If $R$ is 
non-commutative, then there exists a factor subring of $R$ which is of type (a) or (b).

Lemma 4. If $R$ is left (resp. right) s-unital and not right (resp. left) $s$-unital, then $R$ has a factor subring of type $(a)_{1}\left(\right.$ resp. $\left.(a)_{r}\right)$.

\section{Main results}

Theorem 1. Let $R$ be a left s-unital ring satisfying the condition $\left(P_{1}\right)$. Then $R$ is commutative (and conversely).

In order to establish the above theorem, first we prove the following lemma.

Lemma 5. Let $R$ be a ring with unity 1 satisfying any one of the conditions $\left(P_{1}\right)$ and $\left(P_{2}\right)$. Then $R$ has nil commutator ideal.

Proof. Let $R$ satisfy $\left(P_{1}\right)$. Replace $x$ by $1+x$ in $\left(P_{1}\right)$ and subtract $\left(P_{1}\right)$, to get $(1+x)^{m}[x, y]=x^{m}[x, y]$. This is a polynomial identity and $x=e_{12}-e_{22}, y=e_{12}$ in $(G F(p))_{2}$ fail to satisfy this equality. Hence by Lemma $2, R$ has nil commutator ideal.

Using a similar arguments as above, one can establish the result if $R$ satisfies the condition $\left(P_{2}\right)$.

Proof of Theorem 1 . First, consider the ring of type $(a)_{1}$. If $R$ satisfies $\left(P_{1}\right)$, then in $(G F(p))_{2}, p$ a prime, we see that $e_{11}^{m}\left[e_{11}, e_{12}\right]-g\left(e_{12}\right)\left[e_{11}, e_{12}^{2} f\left(e_{12}\right)\right] h\left(e_{12}\right)=$ $e_{12} \neq 0$ for any positive integer $m$ and $f(t), g(t), h(t) \in \mathbb{Z}[t]$. Thus no rings of type $(a)_{1}$ satisfy $\left(P_{1}\right)$.

Next, consider the ring $M_{\sigma}(K)$, a ring of type $(b)$. If $R$ satisfies $\left(P_{1}\right)$, then let $x=\left(\begin{array}{cc}a & 0 \\ 0 & \sigma(a)\end{array}\right)(\sigma(a) \neq a), y=e_{12}$. This yields that

$$
x^{m}[x, y]-g(y)\left[x, y^{2} f(y)\right] h(y)=a^{m}(a-\sigma(a)) e_{12} \neq 0
$$

for all integer $m$ and $f(t), g(t), h(t)$ in $\mathbb{Z}[t]$. Thus $R$ cannot be of type $(b)$.

Further, let $R$ be the ring of type (c). If $R$ satisfies $\left(P_{1}\right)$, then in view of the above, $R$ cannot be of type $(a)_{1}$. Hence in view of Lemma $4, R$ is also right $s$-unital. Thus $R$ is $s$-unital and by [6, Proposition 1], $R$ has unity 1 . Now in view of Lemma 2 and Lemma 5 we see that no rings of type $(c)$ satisfy our assumption.

Finally, suppose that $R$ is a ring of type $(d)$. If $R$ satisfies $\left(P_{1}\right)$, then choose $s, t \in T$ such that $[s, t] \neq 0$. A simple computation shows that

$$
[s, t]=(1+s)^{m}[s+1, t]=g(t)\left[s+1, t^{2} f(t)\right] h(t)=0 .
$$

Hence, we find a contradiction that $[s, t]=0$. This shows that no rings of type $(a)_{1},(b),(c)$ or $(d)$ satisfy $\left(P_{1}\right)$ and in view of Lemma $1, R$ is commutative. 
Corollary 1. Let $R$ be a left s-unital ring in which for every $y$ in $R$ there exist integers $p=p(y) \geq 0, q=q(y) \geq 0, s=s(y)>1$ such that $x^{m}[x, y]=y^{P}\left[x, y^{s}\right] y^{q}$ for all $x$ in $R$, where $m$ is a fixed positive integer. Then $R$ is commutative (and conversely).

In case, if $R$ satisfies $\left(P_{2}\right)$, then we have the following:

Theorem 2. Let $R$ be a right $s$-unital ring satisfying $\left(P_{2}\right)$. Then $R$ is commutative (and conversely).

Proof. Suppose that $R$ is a ring of type $(a)_{r}$. If $R$ satisfies $\left(P_{2}\right)$, then in $(G F(p))_{2}$, $p$ a prime, we find that

$$
\left[e_{22}, e_{12}\right] e_{22}^{m}-g\left(e_{12}\right)\left[e_{22}, e_{12}^{2} f\left(e_{12}\right)\right] h\left(e_{12}\right)=-e_{12} \neq 0
$$

for any positive integer $m$ and $f(t), g(t), h(t) \in \mathbb{Z}[t]$. Accordingly, $R$ cannot be of type $(a)_{r}$. Further, using similar arguments as used to prove Theorem 1 with necessary variations, it can be shown that no rings of type $(b),(c)$ or $(d)$ satisfy our hypothesis. Hence, in view of Lemma $1, R$ is commutative.

Corollary 2. Let $R$ be a right $s$-unital ring in which for every $y$ in $R$ there exist integers $p=p(y) \geq 0, q=q(y) \geq 0, s=s(y)>1$ such that $[x, y] x^{m}=y^{p}\left[x, y^{s}\right] y^{q}$ for all $x$ in $R$, where $m$ is a fixed positive integer. Then $R$ is commutative (and conversely).

Remark. The following example demonstrates that there are non-commutative left (resp. right) $s$-unital rings satisfying $\left(P_{2}\right)$ (resp. $\left.\left(P_{1}\right)\right)$.

Example. Let

$$
\begin{aligned}
R_{1} & =\left\{\left(\begin{array}{ll}
0 & 0 \\
0 & 0
\end{array}\right),\left(\begin{array}{ll}
1 & 0 \\
1 & 0
\end{array}\right),\left(\begin{array}{ll}
0 & 1 \\
0 & 1
\end{array}\right),\left(\begin{array}{ll}
1 & 1 \\
1 & 1
\end{array}\right)\right\} \\
\text { (resp. } R_{2} & \left.=\left\{\left(\begin{array}{ll}
0 & 0 \\
0 & 0
\end{array}\right)\left(\begin{array}{ll}
1 & 1 \\
0 & 0
\end{array}\right)\left(\begin{array}{ll}
0 & 0 \\
1 & 1
\end{array}\right)\left(\begin{array}{ll}
1 & 1 \\
1 & 1
\end{array}\right)\right\}\right)
\end{aligned}
$$

be subring of $2 \times 2$ matrices over $G F(2)$. Then for any fixed positive integers $m, p, q, s>$ $1, R_{1}$ (resp. $R_{2}$ ) satisfies $[x, y] x^{m}-y^{p}\left[x, y^{s}\right] y^{q}=0$ (resp. $x^{m}[x, y]-y^{p} p\left[x, y^{s}\right] y^{q}=0$ ). However, $R_{1}$ (resp. $R_{2}$ ) is a non-commutative left (resp. right) $s$-unital ring.

A careful scrutiny of the proofs of Theorem 1 and Theorem 2 yields that if $R$ is a ring with unity 1 satisfying either of the property $\left(P_{1}^{*}\right)$ or $\left(P_{2}^{*}\right)$, then $R$ has no factor subrings of type $(a)$ or $(b)$. Further, if $R$ satisfies $(C H)$, then in view of Lemma 3 , we get the following:

Theorem 3. Let $R$ be a ring with unity 1 satisfying any one of the conditions $\left(P_{1}^{*}\right)$ and $\left(P_{2}^{*}\right)$. Further, if $R$ satisfies $(C H)$, then $R$ is commutative (and conversely). 
Corollary 3. Suppose that $R$ is a ring with unity 1 in which for every $x, y$ in $R$ there exist integers $m>0, p \geq 0, q \geq 0, s>1$ such that either $x^{m}[x, y]=y^{p}\left[x, y^{s}\right] y$ or $[x, y] x^{m}=y^{p}\left[x, y^{s}\right] y^{q}$. Further, if $R$ satisfies $(C H)$, then $R$ is commutative.

The existence of ring $R$ with $R^{3}=0$ rules out the possible generalization of Theorem 3 for argitary rings. However, if $R$ is a left (resp. right) $s$-unital ring satisfying $\left(P_{1}^{*}\right)$ (resp. $\left.\left(P_{2}^{*}\right)\right)$, then in view of the arguments given in the proofs of Theorem 1 and 2 , we see that no rings of type $(a)_{1}$ (resp. $\left.(a)_{r}\right)$ satisfy $\left(P_{1}^{*}\right)$ (resp. $\left(P_{2}^{*}\right)$ ). Hence, Lemma 4 yields that $R$ is also right (resp. left) $s$-unital. Accordingly, $R$ is $s$ unitial and by [6, Proposition 1], we may assume that $R$ has unity 1 , Hence, using Theorem 3, we get the following

Theorem 4. Let $R$ be a left (resp. right) s-unital ring satisfying $\left(P_{1}^{*}\right.$ ) (resp. $\left.\left(P_{2}^{*}\right)\right)$. Further, if $R$ satisfies $(C H)$, then $R$ is commutative.

\section{Acknowledgement}

The authors are greatly indebted to the refree for his valuable suggestions.

\section{References}

[1] H. A. S. Abu-Jabal and V. Perić, "Commutativity of $s$-unital rings through a streb result," Rad. Mat., 7 (1991), 73-92.

[2] M. Ashraf and M. A. Quadri, "On commutativity of associative rings," Bull. Austral. Math. Soc., 38 (1988), 267-271.

[3] M. Ashraf, M. A. Quadri and V. W. Jacob, "On commutativity of right s-unital rings," Riv. Mat. Univ. Parma, (to appear).

[4] H. E. Bell, "On some commutativity theorems of Herstein," Arch. Math. (Basel), 24 (1973), 34-38.

[5] H. E. Bell, "On power maps and ring commutativity," Canad. Math. Bull., 21 (1978), 399-404.

[6] Y. Hirano, Y. Kobayashi and H. Tominaga, "Some polynomial identities and commutativity of s-unital rings," Math. J. Okayama Univ., 24 (1982), 7-13.

[7] T. P. Kezlan, "A note on commutativity of semi prime PI-rings," Math. Japonica, 27 (1982), 267-268.

[8] H. Komatsu and H. Tominaga, "Chacron's condition and commutativity theorems," Math. J. Oka.yama Univ., 31 (1989), 101-120.

[9] H. Komatsu and H. Tominaga, "Some commutativity theorems for left $s$-unital rings," Results in Math., 15 (1989), 335-342.

[10] H. Komatsu, T. Nishinaka and H. Tominaga, "On commutativity of rings," Rad. Mat., 6 (1990), 303-311.

[11] E. Psomopoulos, "Commutativity theorem for rings and groups with constraints on commutators," Internat. J. Math. \& Math. Sci., 7 (1984), 513-517.

[12] W. Streb, "Zur Struktur nichtkommutativer Ringe," Math. J. Okayama Univ., 31 (1989), $135-140$.

Department of Mathematics, Faculty of Science, King Abdul Aziz University, P.O. Box 31464, Jeddah 21497, Saudi Arabia.

Department of Mathematics, Aligarh Muslim University, Aligarh - 202002 - India. 